\title{
Heating of a Trapped Ion Induced by Dielectric Materials
}

\author{
Markus Teller@, ${ }^{1}$ Dario A. Fioretto, ${ }^{1}$ Philip C. Holz $\odot,{ }^{1,2}$ Philipp Schindler, ${ }^{1}$ Viktor Messerer, ${ }^{1}$ Klemens Schüppert $\odot,{ }^{1}$ \\ Yueyang Zou, ${ }^{1}$ Rainer Blatt, ${ }^{1,3}$ John Chiaverini, ${ }^{4,5}$ Jeremy Sage,,${ }^{4,5}$ and Tracy E. Northup $\circledast^{1, *}$ \\ ${ }^{1}$ Institut für Experimentalphysik, Universität Innsbruck, Technikerstrasse 25, 6020 Innsbruck, Austria \\ ${ }^{2}$ Alpine Quantum Technologies GmbH, Technikerstrasse 17/1, 6020 Innsbruck, Austria \\ ${ }^{3}$ Institut für Quantenoptik und Quanteninformation, Österreichische Akademie der Wissenschaften, \\ Technikerstrasse 21 A, 6020 Innsbruck, Austria \\ ${ }^{4}$ Lincoln Laboratory, Massachusetts Institute of Technology, Lexington, Massachusetts 02421, USA \\ ${ }^{5}$ Massachusetts Institute of Technology, Cambridge, Massachusetts 02139, USA
}

(Received 26 March 2021; accepted 17 May 2021; published 11 June 2021)

\begin{abstract}
Electric-field noise due to surfaces disturbs the motion of nearby trapped ions, compromising the fidelity of gate operations that are the basis for quantum computing algorithms. We present a method that predicts the effect of dielectric materials on the ion's motion. Such dielectrics are integral components of ion traps. Quantitative agreement is found between a model with no free parameters and measurements of a trapped ion in proximity to dielectric mirrors. We expect that this approach can be used to optimize the design of ion-trap-based quantum computers and network nodes.
\end{abstract}

DOI: 10.1103/PhysRevLett.126.230505

In a trapped-ion quantum computer, entangling gate operations rely on a shared motional degree of freedom $[1,2]$. For high-fidelity entanglement, as is now standard in prototype quantum computing experiments [3-5], the ions' motional state must be preserved during a gate operation $[2,6]$. This requirement may be compromised by electricfield fluctuations, which in a trapped-ion experiment couple to the motion of the ions and increase their temperature [6,7]. Electric-field noise presents a challenge in particular for scaling up the number of quantum bits while maintaining high-fidelity gate operations $[8,9]$.

While electric-field noise is a topic of much current research, we lack a complete understanding of the physical mechanisms behind it [8]. In recent years, Johnson noise, patch potentials, and surface adsorbates have been investigated as noise sources [10-13]. It has been pointed out that dielectric materials may be a significant noise source even for thin layers, such as in the case of surface oxides and adsorbates [14,15].

Here we present and test a method that predicts ion motional heating due to the proximity to a dielectric. We take advantage of an experimental setup with integrated optics whose distance to a trapped ion can be varied, in which the dielectric makes the dominant contribution to electric-field noise. Our measurements show quantitative

Published by the American Physical Society under the terms of the Creative Commons Attribution 4.0 International license. Further distribution of this work must maintain attribution to the author(s) and the published article's title, journal citation, and DOI. agreement with our predictive model, which has no free parameters. Since dielectrics are ubiquitous in ion traps as integrated optics [16-23], as trap substrates [24,25], and as surface contamination, we anticipate that our approach will enable a broad assessment of the contribution of dielectrics to ion heating in existing traps. Moreover, it will also allow researchers to minimize the motional heating of future traps during the design and fabrication phases, an important step for the development of quantum computers and for quantum network nodes, which require efficient optical interfaces.

First, we summarize the formalism for predicting the electric-field noise at the position of an ion due to a dielectric with arbitrary geometry. The formalism is based on the derivation of Ref. [14], which considers the case of infinite planes, and which we have adapted for finite volumes of arbitrary shape; a sketch of the key elements is depicted in Fig. 1. We consider a charged particle with mass $m$ that oscillates with frequency $\omega$ in vacuum (permittivity $\epsilon_{0}$ ) near a dielectric material. We assume that the distance $d$ between the dielectric and the particle is significantly larger than both the oscillation amplitude parallel to the surface, $\delta x$, and the oscillation amplitude perpendicular to the surface, $\delta z$. We use $\delta \zeta$ to refer to one of the two amplitudes: $\delta \zeta \in\{\delta x, \delta z\}$ and consider the particle's motion along the axis $\zeta \in\{x, z\}$. The dielectric material is characterized by its complex permittivity $\epsilon_{1}=\epsilon_{0} \varepsilon_{r}(1+i \tan \delta)$, with $\tan \delta$ the loss tangent and $\varepsilon_{r}$ the relative permittivity. The particle, which has charge $q$, generates an electric field $\mathbf{E}_{\zeta}=\mathbf{E}_{0}+\operatorname{Re}\left[\mathbf{E}_{1, \zeta} e^{i \omega t}\right]$, which leads to a displacement field $\mathbf{D}_{\zeta}=\epsilon_{1} \mathbf{E}_{\zeta}=\mathbf{D}_{0}+$ $\operatorname{Re}\left[\mathbf{D}_{1, \zeta} e^{i \omega t}\right]$ inside the dielectric. Note that $\mathbf{E}_{0}$ and $\mathbf{D}_{0}$ 


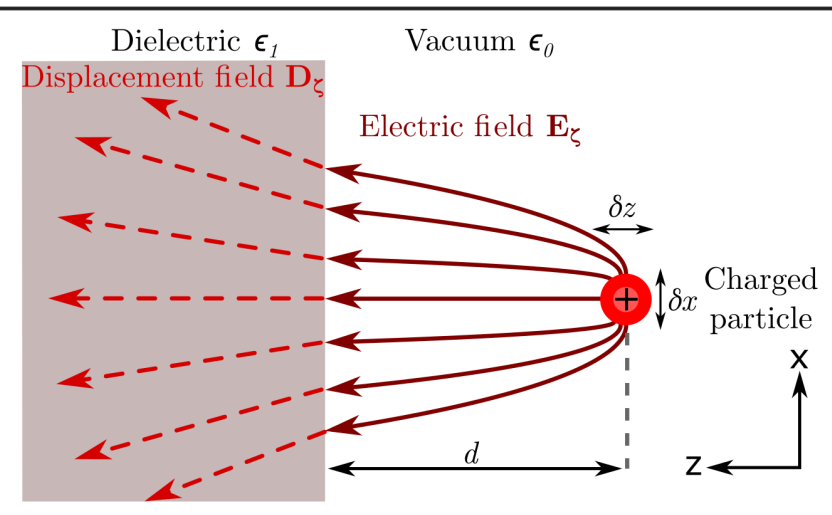

FIG. 1. A charged particle is placed a distance $d$ from a dielectric material with permittivity $\epsilon_{1}$. The particle oscillates in vacuum (permittivity $\epsilon_{0}$ ) with amplitude $\delta \zeta \ll d$, with $\delta \zeta \in\{\delta x, \delta z\}$. The charge generates an electric field $\mathbf{E}_{\zeta}$ outside the dielectric and a displacement field $\mathbf{D}_{\zeta}$ inside.

correspond to the fields arising from the average particle position, whereas $\mathbf{E}_{1, \zeta}$ and $\mathbf{D}_{1, \zeta}$ are due to the motion of the particle with amplitude $\delta \zeta$.

Losses inside the dielectric material are described by the power loss of the displacement field as [14]

$$
\left\langle P_{\text {loss }}\right\rangle=\int_{V} \frac{1}{2} \operatorname{Re}\left\{-i \omega \mathbf{E}_{1, \zeta}^{*} \cdot \mathbf{D}_{1, \zeta}\right\}=\frac{\omega}{2} \epsilon_{0} \varepsilon_{r} \tan \delta \int_{V}\left|\mathbf{E}_{1, \zeta}\right|^{2} .
$$

Here, the power loss is averaged over an oscillation period of the particle, and the integral is evaluated over the volume $V$ of the dielectric. The power loss is related to the particle motion via [14]

$$
\left\langle P_{\text {loss }}\right\rangle=\frac{1}{2} m \omega^{2} \delta \zeta^{2} \operatorname{Re}\{\gamma[\omega]\},
$$

where $\gamma$ denotes a frequency-dependent damping term.

By combining Eqs. (1) and (2) with the fluctuationdissipation theorem as expressed in Ref. [14], we obtain an expression for the power spectral density

$$
S_{E}(\omega)=\frac{4 k_{B} T}{\delta \zeta^{2} q^{2} \omega} \epsilon_{0} \varepsilon_{r} \tan \delta \int_{V}\left|\mathbf{E}_{1, \zeta}\right|^{2}
$$

of the electric-field fluctuations emerging from the dielectric at the particle position; these fluctuations, at the particle's oscillation frequency, are along the axis of the particle motion. Here, $k_{B}$ is the Boltzmann constant and $T$ the temperature of the dielectric. Equation (3) thus relates energy dissipation inside the dielectric, quantified by $\mathbf{E}_{1, \zeta}$, to the electric field fluctuations originating from the dielectric. The equation can be evaluated numerically by means of finite-element analysis (FEA) software that solves Maxwell's equations for arbitrary geometries.
We now turn to the experimental setup used to test our model, adapted from a setup designed for quantum networking [26] and depicted in Fig. 2. A ${ }^{40} \mathrm{Ca}^{+}$ion, trapped in a three-dimensional linear Paul trap at room temperature, is located in the center of a fiber-based optical cavity [26,27]. The trap has a radial ion-electrode distance of $250 \mu \mathrm{m}$. For the measurements presented in this Letter, the axial secular frequency $\omega_{z}$ is varied from $2 \pi \times 0.5$ to $2 \pi \times 1.6 \mathrm{MHz}$, while the radial secular frequencies are kept at $\omega_{x, y} \approx 2 \pi \times 3.3 \mathrm{MHz}$. The fiber-based optical cavity is formed by two highly reflective mirrors imprinted on the facets of a photonic-crystal fiber and a multimode fiber [28]. The mirror on the photonic-crystal fiber is composed of 41 alternating layers of ion-beam-sputtered $\mathrm{SiO}_{2}$ and $\mathrm{Ta}_{2} \mathrm{O}_{5}$; the mirror on the multimode fiber is composed of 47 alternating layers. For both fiber mirrors, the top layer is $\mathrm{SiO}_{2}$. The thickness of each layer corresponds to a quarter of the operating cavity wavelength of $\lambda=854 \mathrm{~nm}$. The fiber-cavity length is determined from measurements of the free spectral range, which have an uncertainty of $8 \mu \mathrm{m}$. The length can be varied from 500 to $1200 \mu \mathrm{m}$ by means of piezo stages with a resolution of $1 \mathrm{~nm}$ for relative fiber movements. We have insufficient optical access to determine the absolute position of the ion precisely with respect to each fiber. The ion-fiber distance $d$ is estimated to be half the fiber-fiber distance and therefore has a systematic uncertainty, determined from the Rayleigh length of the cavity to be $55 \mu \mathrm{m}$ [26].

Using the formalism described above, we predict the electric-field noise originating from the dielectric in our experimental apparatus. In our FEA simulations, each fiber is modeled as a $300 \mu \mathrm{m}$-long cylinder with a diameter of $230 \mu \mathrm{m}$. Each layer of the mirror coating is modeled as a cylinder with $250 \mathrm{~nm}$ thickness and the same diameter as the fiber. The layer thickness of $250 \mathrm{~nm}$ is the closest value to $\lambda / 4$ that we can resolve with the FEA simulation mesh. The fiber is assumed to be at room temperature, $T=300 \mathrm{~K}$. For the relative permittivities of $\mathrm{SiO}_{2}$ and $\mathrm{Ta}_{2} \mathrm{O}_{5}$, we use literature values of $\varepsilon_{r, S}=3.9$, with a loss tangent $\tan \delta_{S}=1.3 \times 10^{-3}$, and $\varepsilon_{r, T}=22$, with $\tan \delta_{T}=$ $7 \times 10^{-3}[29,30]$, respectively.

We evaluate Eq. (3) for each mirror-coated fiber separately and sum the two noise contributions. We take the calcium ion to be the point charge of Fig. 1, and our task is to solve for the stationary field $\mathbf{E}_{0}$ inside the dielectric of Fig. 2. The axes for the ion's radial and axial oscillations are shown in Figs. 1 and 2. By displacing the particle by $\delta \zeta=5 \mu \mathrm{m}$ along a given axis and solving again for the field $\mathbf{E}_{\zeta}$, we determine the field $\mathbf{E}_{1, \zeta}=\mathbf{E}_{\zeta}-\mathbf{E}_{0}$ due to the oscillation of the particle along that axis. Note that the axis of the particle displacement defines the axis of the simulated electric-field noise. As long as the condition $\delta \zeta \ll d$ is fulfilled, the choice of $5 \mu \mathrm{m}$ for $\delta \zeta$ is somewhat arbitrary since in Eq. (3), the integral of $\left|\mathbf{E}_{1, \zeta}\right|^{2}$ is divided by $\delta \zeta^{2}$, and thus $S_{E}(\omega)$ is independent of the displacement 


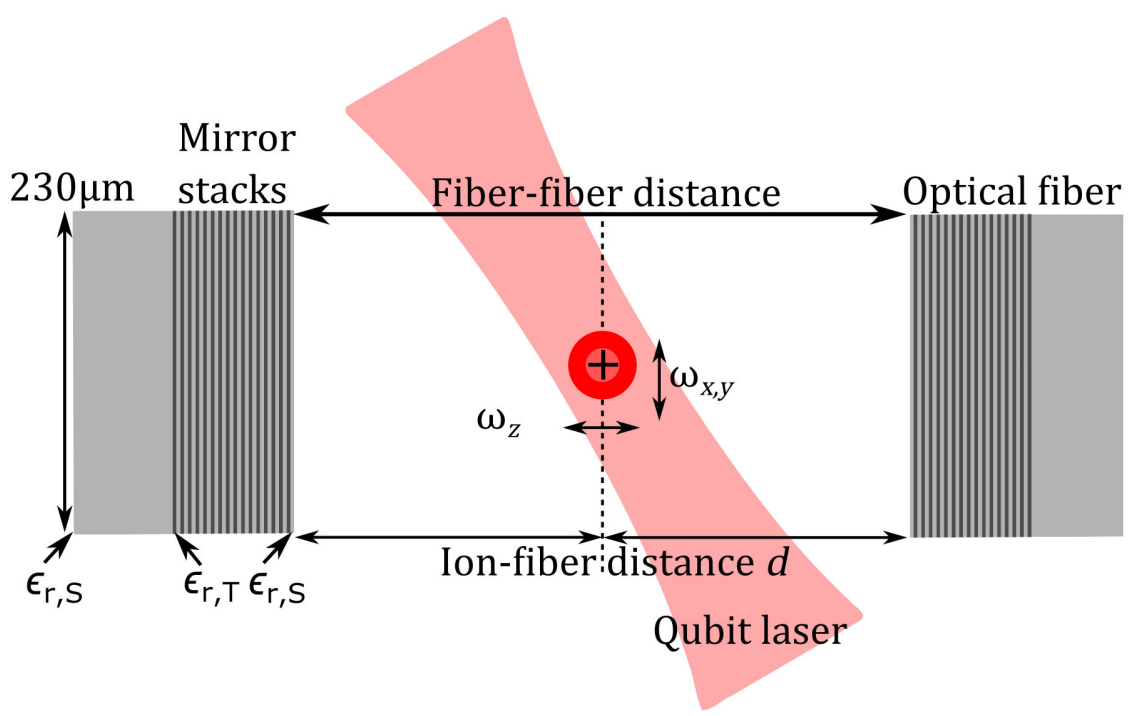

FIG. 2. A trapped ion with axial and radial frequencies $\omega_{z}$ and $\omega_{x, y}$ is placed in the vicinity of two optical fibers. The facets of the optical fibers are laser ablated and coated with dielectric stacks, composed of alternating layers of $\mathrm{SiO}_{2}$ and $\mathrm{Ta}_{2} \mathrm{O}_{5}$, with respective permittivities $\varepsilon_{r, S}$ and $\varepsilon_{r, T}$. For our simulations, we neglect the mirror radii of curvature, which are $312(5) \mu \mathrm{m}$ for the photonic-crystal fiber and $318(5) \mu \mathrm{m}$ for the multimode fiber.

value. However, in the FEA simulation, $\delta \zeta$ has to be small enough to avoid effects from the borders of the simulated geometry yet larger than the meshing resolution of our simulated geometry. We found the simulated electric-field noise to be independent of $\delta \zeta$ for displacements between 5 and $25 \mu \mathrm{m}$ for all ion-dielectric separations considered in this work.

In the following experiments, we investigate whether the rate at which the ion's temperature increases is supported by our predictions of the electric-field noise emerging from the mirror-coated fibers. We measure the motional heating rate using the decay of carrier Rabi oscillations due to the Debye-Waller coupling [31-34]. After Doppler cooling and preparation of the ion in the $\left|4^{2} S_{1 / 2}, m_{j}=-1 / 2\right\rangle$ state, we wait for a time $t$ and then excite the $\left|4^{2} S_{1 / 2}, m_{j}=-1 / 2\right\rangle$ to $\left|3^{2} D_{5 / 2}, m_{j}=-1 / 2\right\rangle^{40} \mathrm{Ca}^{+}$transition using a narrow-linewidth laser with wavelength $\lambda=729 \mathrm{~nm}$. The laser beam overlaps with the ion's radial and axial modes of motion and therefore probes the ion's temperature along all three axes simultaneously [35]. From a fit of Rabi oscillations in which each data point is weighted according to the quantum projection noise, we extract the parameter

$$
\beta=\sum_{i} \bar{n}_{i} \eta_{i}^{2}
$$

where $\bar{n}_{i}$ is the mean phonon number along the motional axis $i \in\{x, y, z\}$ and $\eta_{i}=2 \pi / \lambda \sqrt{\hbar / 2 m \omega_{i}} \cos \left(\phi_{i}\right)$ denotes the effective Lamb-Dicke factor of each mode, with $\phi_{i}$ the angle between the mode axis and the wave vector [31]. We determine $\beta$ for several values of $t$ and deduce $\dot{\beta}$ from a linear fit. We identify $\dot{\beta}$ as a weighted sum of the heating rates $\dot{\bar{n}}_{i}$ along the three motional axes: $\dot{\beta}=\sum_{i} \dot{\bar{n}}_{i} \eta_{i}^{2}$. The uncertainty of $\dot{\beta}$ corresponds to the standard deviation of this fit.

In order to compare experimental observations to the predictions of our model, we calculate the heating rate along each motional axis that results from the simulated electric-field noise [8]:

$$
\dot{\bar{n}}_{i}=\frac{q^{2}}{4 m \hbar \omega} S_{E}\left(\omega_{i}\right)
$$

Thus, we predict the heating rates for all three axes separately, allowing us to determine $\dot{\beta}$.

For our experimental geometry, the axial contribution to the heating rate dominates by more than 1 order of magnitude [36]. Thus, the heating rate along the $z$ axis in the system is approximately equal to (though smaller than) $\dot{\beta} / \eta_{z}^{2}$. For easier comparison with observations of other experimental platforms, which typically consider heating of just one mode at a time, we define $\dot{\bar{n}}=\dot{\beta} / \eta_{z}^{2}$ as a projection onto the $z$ axis.

We first study the frequency response of the heating rate. For these measurements, we vary $\omega_{z}$ for two ion-fiber separations of 450 and $600 \mu \mathrm{m}$. We choose the values of $\omega_{z}$ such that the motional sidebands of the $\mid 4^{2} S_{1 / 2}, m_{j}=$ $-1 / 2\rangle$ to $\left|3^{2} D_{5 / 2}, m_{j}=-1 / 2\right\rangle$ transition do not overlap with transitions between other Zeeman states. In Fig. 3, we plot measured data together with predictions from simulations based on literature values for the dielectric material properties, with no free parameters. Over a frequency range from $2 \pi \times 0.5 \mathrm{MHz}$ to $2 \pi \times 1.5 \mathrm{MHz}$, the heating rate varies by an order of magnitude for both separations, which 


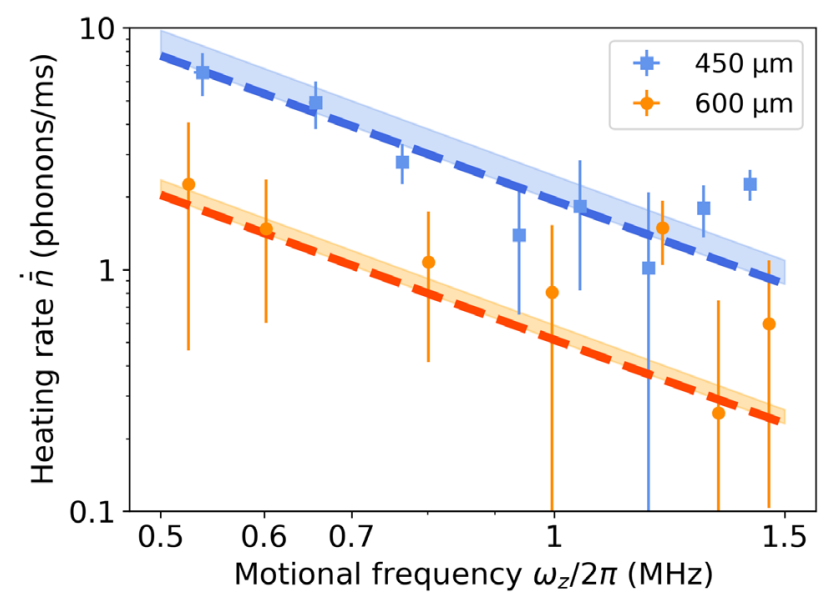

FIG. 3. The heating rate as a function of axial frequency for iondielectric separations of 450 (squares) and $600 \mu \mathrm{m}$ (circles). The dashed lines represent the predictions based on the literature values, and light-orange and light-blue areas show systematic uncertainties of the ion-fiber distance. The systematic uncertainties are determined from predictions that consider one fiber $55 \mu \mathrm{m}$ closer to the ion and the other fiber $55 \mu \mathrm{m}$ more distant. For both versions of such an asymmetric placement, the heating rates are larger than for the symmetric case, and thus, the systematic uncertainty is single sided.

is captured accurately by our FEA-based noise estimation. We calculate a reduced chi-square value of $\chi_{\nu}^{2}=1.39$ for experiment and predictions, for both datasets combined. Given this agreement, an interesting application is that our method can be employed to determine the loss tangent of unknown dielectric materials [36].

Second, we measure the distance scaling of the heating rate, varying $d$ while keeping the ion position fixed within $25 \mu \mathrm{m}$ using a CCD camera image as reference. For each distance, we measure the axial trap frequency $\omega_{z} / 2 \pi$, which ranges from 1.300(8) to 1.696(3) MHz. This measurement is necessary as the trap potential changes with $d$ due to surface charges on the dielectric. In Fig 4, we show the experimental data and the predicted heating rates from our model without free parameters. The predicted rates are plotted as discrete values evaluated for each measured ionfiber separation and trap frequency. Additionally, we fit the eight simulated electric-field-noise values with a power-law function $S_{E}=A d^{-\alpha}$ [36]. The radial and axial scaling parameters are determined to be $\alpha_{\mathrm{rad}}=4.413(6)$ and $\alpha_{\mathrm{ax}}=4.016(6)$. The divergence of these parameters from the $1 / d^{3}$ scaling of an infinite dielectric plane underscores the necessity of our method for finite geometries [42]. To visualize the scaling for a single trap frequency, we plot the heating rate based on the fitted electric-field noise as a continuous function for $\omega_{z}=2 \pi \times 1.6 \mathrm{MHz}$.

For the full set of data shown in Fig. 4, from $d=250$ to $d=600 \mu \mathrm{m}$, we calculate $\chi_{\nu}^{2}=2.57$ for the measurements and the model. Excluding the point corresponding to the shortest distance of $d=250 \mu \mathrm{m}$ results in a value of

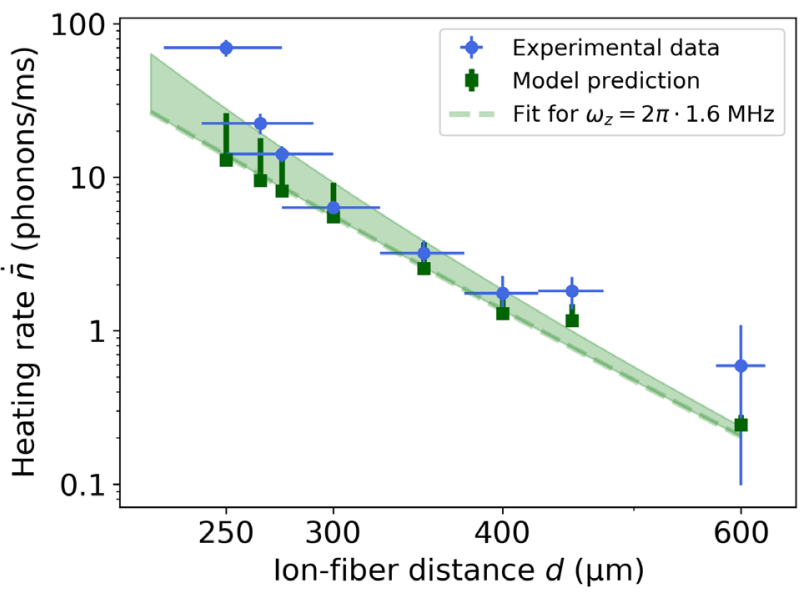

FIG. 4. The heating rate as a function of ion-fiber distance. The green points represent the prediction with error bars corresponding to the systematic uncertainty of the ion-fiber distance. The dashed line depicts a fit for a trap frequency of $\omega_{z}=2 \pi \times 1.6 \mathrm{MHz}$, and the respective systematic uncertainty is illustrated in light green. The asymmetry of the systematic uncertainty is discussed in the caption of Fig. 3.

$\chi_{\nu}^{2}=0.86$, demonstrating strong agreement. At this short distance, the axial trapping potential is significantly influenced by inhomogeneously distributed surface charges, which, however, are not considered in the simulations $[36,43]$. Potentially, more information could be extracted by measuring a frequency scaling similar to that of Fig. 3 . However, below $d=300 \mu \mathrm{m}$, the ion is observed to be unstable for $\omega_{\mathrm{z}} \leq 2 \pi \times 1.5 \mathrm{MHz}$, impeding such a measurement.

In summary, we have presented a new FEA-based method to predict electric field noise from dielectrics with arbitrary geometry. We have taken advantage of an ion-trap experiment with a movable dielectric element in order to test this method. The measured heating rates are attributed to the influence of the bulk dielectric as they are up to 3 orders of magnitude larger than reference values measured before the trap was integrated with the cavity: 13(3) phonons/s for the axial mode of motion at a frequency of $2 \pi \times 1.5 \mathrm{MHz}$ and $\{26(6), 32(8)\}$ phonons/s for the radial modes at frequencies of $2 \pi \times 3.2,3.4 \mathrm{MHz}$ [26]. While other sources of technical noise may be present, we do not expect the heating rates due to these sources to scale with the distance to the fiber. The magnitude of the electric-field noise predicted by our model without free parameters agrees with the measured data for a range of motional frequencies and ion-dielectric separations. The analysis for the ion-dielectric separation of $250 \mu \mathrm{m}$ and below will require further study.

Here we have focused on the effects of a bulk dielectric, but we emphasize that the contributions of dielectric materials to heating in ion traps may be a much more common phenomenon. As an example, we predict the influence of an $\mathrm{SiO}_{2}$ cylinder with radius of $10 \mu \mathrm{m}$ and a 
thickness of only one nanometer. Such a structure could appear as surface contamination during the fabrication of an ion trap. Assuming an ion-dielectric separation of $50 \mu \mathrm{m}$ and trap frequencies of $\omega=2 \pi \times 1 \mathrm{MHz}$ for all three modes of a ${ }^{40} \mathrm{Ca}^{+}$ion, we find heating rates of 127 phonons/s for the radial modes and 170 phonons/s for the axial mode of motion. We thus see that nanoscale patches of surface oxides may lead to significant heating when compared to the target rates for scalable ion-trap quantum computing and communication platforms [44]. Note that in this model, the dielectric is assumed to be at room temperature and surface effects such as surface charges are not considered.

Our prediction method can be used to systematically optimize the design and fabrication of future trapped-ion setups, as the electrode geometry and materials_-including contaminant or oxide layers - can be adjusted in the FEA simulations to suppress heating effects due to dielectrics. Finally, we note that these results are also relevant for devices such as nitrogen-vacancy centers $[45,46]$ and donor spins in silicon [47] that are disturbed by environmental noise due to nearby surfaces.

All data presented and discussed in this Letter are available at [48].

We thank Carsten Henkel for his feedback on the infinite plane model and for his suggestion to use the fluctuationdissipation theorem to simulate heating rates. We thank Brenda Rubenstein and Yuan Liu for interesting and fruitful discussions. This work was supported by the European Union's Horizon 2020 research and innovation program under Grant Agreement No. 820445 (Quantum Internet Alliance), by the U.S. Army Research Laboratory under Cooperative Agreement No. W911NF-15-2-0060, and by the Austrian Science Fund (FWF) under Project No. F 7109. P. H. acknowledges support from the European Union's Horizon 2020 research and innovation programme under Grant Agreement No. 801285 (PIEDMONS). P.S. acknowledges support from the Austrian Research Promotion Agency (FFG) contract 872766. We also acknowledge support from the Institut für Quanteninformation $\mathrm{GmbH}$. This material is based upon work supported by the Department of Defense under Air Force Contract No. FA8702-15-D-0001. Any opinions, findings, conclusions, or recommendations expressed in this material are those of the authors and do not necessarily reflect the views of the Department of Defense.

*Corresponding author tracy.northup@uibk.ac.at

[1] J. I. Cirac and P. Zoller, Quantum Computations with Cold Trapped Ions, Phys. Rev. Lett. 74, 4091 (1995).

[2] K. Mølmer and A. Sørensen, Multiparticle Entanglement of Hot Trapped Ions, Phys. Rev. Lett. 82, 1835 (1999).
[3] F. Schmidt-Kaler, H. Häffner, M. Riebe, S. Gulde, G. P. T. Lancaster, T. Deuschle, C. Becher, C. F. Roos, J. Eschner, and R. Blatt, Realization of the Cirac-Zoller controlled-NOT quantum gate, Nature (London) 422, 408 (2003).

[4] P. C. Haljan, K.-A. Brickman, L. Deslauriers, P. J. Lee, and C. Monroe, Spin-Dependent Forces on Trapped Ions for Phase-Stable Quantum Gates and Entangled States of Spin and Motion, Phys. Rev. Lett. 94, 153602 (2005).

[5] A. Erhard, J. J. Wallman, L. Postler, M. Meth, R. Stricker, E. A. Martinez, P. Schindler, T. Monz, J. Emerson, and R. Blatt, Characterizing large-scale quantum computers via cycle benchmarking, Nat. Commun. 10, 5347 (2019).

[6] C. Monroe, D. M. Meekhof, B. E. King, S. R. Jefferts, W. M. Itano, D. J. Wineland, and P. Gould, ResolvedSideband Raman Cooling of a Bound Atom to the 3d Zero-Point Energy, Phys. Rev. Lett. 75, 4011 (1995).

[7] Q. A. Turchette, D. Kielpinski, B. E. King, D. Leibfried, D. M. Meekhof, C. J. Myatt, M. A. Rowe, C. A. Sackett, C. S. Wood, W. M. Itano, C. Monroe, and D. J. Wineland, Heating of trapped ions from the quantum ground state, Phys. Rev. A 61, 063418 (2000).

[8] M. Brownnutt, M. Kumph, P. Rabl, and R. Blatt, Ion-trap measurements of electric-field noise near surfaces, Rev. Mod. Phys. 87, 1419 (2015).

[9] K. R. Brown, J. Chiaverini, J. Sage, and H. Häffner, Materials challenges for trapped-ion quantum computers, arXiv:2009.00568.

[10] N. Daniilidis, S. Gerber, G. Bolloten, M. Ramm, A. Ransford, E. Ulin-Avila, I. Talukdar, and H. Häffner, Surface noise analysis using a single-ion sensor, Phys. Rev. B 89, 245435 (2014).

[11] C. Noel, M. Berlin-Udi, C. Matthiesen, J. Yu, Y. Zhou, V. Lordi, and H. Häffner, Electric-field noise from thermally activated fluctuators in a surface ion trap, Phys. Rev. A 99, 063427 (2019).

[12] G. H. Low, P.F. Herskind, and I. L. Chuang, Finitegeometry models of electric field noise from patch potentials in ion traps, Phys. Rev. A 84, 053425 (2011).

[13] A. Safavi-Naini, E. Kim, P. F. Weck, P. Rabl, and H. R. Sadeghpour, Influence of monolayer contamination on electric-field-noise heating in ion traps, Phys. Rev. A 87, 023421 (2013).

[14] M. Kumph, C. Henkel, P. Rabl, M. Brownnutt, and R. Blatt, Electric-field noise above a thin dielectric layer on metal electrodes, New J. Phys. 18, 023020 (2016).

[15] Y. Liu and B. M. Rubenstein (private communication).

[16] G. R. Guthöhrlein, M. Keller, K. Hayasaka, W. Lange, and $\mathrm{H}$. Walther, A single ion as a nanoscopic probe of an optical field, Nature (London) 414, 49 (2001).

[17] J. Eschner, C. Raab, F. Schmidt-Kaler, and R. Blatt, Light interference from single atoms and their mirror images, Nature (London) 413, 495 (2001).

[18] A. B. Mundt, A. Kreuter, C. Becher, D. Leibfried, J. Eschner, F. Schmidt-Kaler, and R. Blatt, Coupling a Single Atomic Quantum Bit to a High Finesse Optical Cavity, Phys. Rev. Lett. 89, 103001 (2002).

[19] A. P. VanDevender, Y. Colombe, J. Amini, D. Leibfried, and D. J. Wineland, Efficient Fiber Optic Detection of Trapped Ion Fluorescence, Phys. Rev. Lett. 105, 023001 (2010). 
[20] T. H. Kim, P. F. Herskind, and I. L. Chuang, Surfaceelectrode ion trap with integrated light source, Appl. Phys. Lett. 98, 214103 (2011).

[21] M. Steiner, H. M. Meyer, C. Deutsch, J. Reichel, and M. Köhl, Single Ion Coupled to an Optical Fiber Cavity, Phys. Rev. Lett. 110, 043003 (2013).

[22] R. J. Niffenegger, J. Stuart, C. Sorace-Agaskar, D. Kharas, S. Bramhavar, C. D. Bruzewicz, W. Loh, R. T. Maxson, R. McConnell, D. Reens, G. N. West, J. M. Sage, and J. Chiaverini, Integrated multi-wavelength control of an ion qubit, Nature (London) 586, 538 (2020).

[23] K. K. Mehta, C. Zhang, M. Malinowski, T.-L. Nguyen, M. Stadler, and J. P. Home, Integrated optical multi-ion quantum logic, Nature (London) 586, 533 (2020).

[24] M. D. Hughes, B. Lekitsch, J. A. Broersma, and W. K. Hensinger, Microfabricated ion traps, Contemp. Phys. 52, 505 (2011).

[25] D.-I. D. Cho, S. Hong, M. Lee, and T. Kim, A review of silicon microfabricated ion traps for quantum information processing, Micro Nano Sys. Lett. 3, 2 (2015).

[26] M. Teller, V. Messerer, K. Schüppert, Y. Zou, D. A. Fioretto, P. C. Holz, R. Blatt, and T. E. Northup (unpublished).

[27] T. Steinmetz, Y. Colombe, D. Hunger, T. W. Hänsch, A. Balocchi, R. J. Warburton, and J. Reichel, Stable fiber-based Fabry-Pérot cavity, Appl. Phys. Lett. 89, 111110 (2006).

[28] K. Ott, S. Garcia, R. Kohlhaas, K. Schüppert, P. Rosenbusch, R. Long, and J. Reichel, Millimeter-long Fabry-Pérot cavities, Opt. Express 24, 9839 (2016).

[29] J. Pushkar and E. J. Rymaszewski, Thin-Film Capacitors for Packaged Electronics (Springer US, New York, 2004).

[30] J.-Y. Kim, A. Garg, E. Rymaszewski, and T.-M. Lu, High frequency response of amorphous tantalum oxide thin films, IEEE Trans. Compon. Packag. Technol. 24, 526 (2001).

[31] C. F. Roos, Controlling the quantum state of trapped ions, Ph.D. thesis, University of Innsbruck, 2000.

[32] M. A. Rowej, A. Ben-Kish, B. Demarco, D. Leibfried, V. Meyer, J. Beall, J. Britton, J. Hughes, W. M. Itano, B. Jelenković, C. Langer, T. Rosenband, and D. J. Wineland, Transport of quantum states and separation of ions in a dual rf ion trap, Quantum Inf. Comput. 2, 257 (2002).

[33] H. Häffner, C. Roos, and R. Blatt, Quantum computing with trapped ions, Phys. Rep. 469, 155 (2008).

[34] D. An, C. Matthiesen, E. Urban, and H. Häffner, Distance scaling and polarization of electric-field noise in a surface ion trap, Phys. Rev. A 100, 063405 (2019).

[35] D. J. Wineland, C. Monroe, W. M. Itano, D. Leibfried, B. E. King, and D. M. Meekhof, Experimental issues in coherent quantum-state manipulation of trapped atomic ions, J. Res. Natl. Inst. Stand. Technol. 103, 259 (1998).
[36] See Supplemental Material at http://link.aps.org/ supplemental/10.1103/PhysRevLett.126.230505 for more details on fitting of the distance scaling, testing the simulation method with an infinite plane, and determining the loss tangent from the frequency scaling, as well as for further discussion and insights on Fig. 4, including a table of axial trap frequencies corresponding to the data and Refs. [37-41].

[37] J. M. Wylie and J. E. Sipe, Quantum electrodynamics near an interface, Phys. Rev. A 30, 1185 (1984).

[38] M.-C. Wang, C.-Y. Chen, C.-S. Hsi, and N.-C. Wu, Influence of deposition parameters on the dielectric properties of rf magnetron sputtered $\mathrm{Ba}\left(\mathrm{Zr}_{\mathrm{x}} \mathrm{Ti}_{1-\mathrm{x}}\right) \mathrm{O}_{3}$ thin films, J. Eur. Ceram. Soc. 23, 2307 (2003).

[39] L. F. Chen, C. K. Ong, C. P. Neo, V. V. Varadan, and V. K. Varadan, Microwave Electronics (John Wiley \& Sons, Ltd., Chichester, 2004), Chap. 12, pp. 492-530.

[40] L. F. Chen, C. K. Ong, C. P. Neo, V. V. Varadan, and V. K. Varadan, Microwave Electronics (John Wiley \& Sons, Ltd., Chichester, 2004), Chap. 2, pp. 37-139.

[41] K. K. Mehta, C. D. Bruzewicz, R. McConnell, R. J. Ram, J. M. Sage, and J. Chiaverini, Integrated optical addressing of an ion qubit, Nat. Nanotechnol. 11, 1066 (2016).

[42] C. Henkel, S. Pötting, and M. Wilkens, Loss and heating of particles in small and noisy traps, Appl. Phys. B 69, 379 (1999).

[43] F. R. Ong, K. Schüppert, P. Jobez, M. Teller, B. Ames, D. A. Fioretto, K. Friebe, M. Lee, Y. Colombe, R. Blatt, and T. E. Northup, Probing surface charge densities on optical fibers with a trapped ion, New J. Phys. 22, 063018 (2020).

[44] A. Bermudez, X. Xu, R. Nigmatullin, J. O'Gorman, V. Negnevitsky, P. Schindler, T. Monz, U. G. Poschinger, C. Hempel, J. Home, F. Schmidt-Kaler, M. Biercuk, R. Blatt, S. Benjamin, and M. Müller, Assessing the Progress of Trapped-Ion Processors Towards Fault-Tolerant Quantum Computation, Phys. Rev. X 7, 041061 (2017).

[45] B. A. Myers, A. Ariyaratne, and A. C. B. Jayich, DoubleQuantum Spin-Relaxation Limits to Coherence of NearSurface Nitrogen-Vacancy Centers, Phys. Rev. Lett. 118, 197201 (2017).

[46] P. Chrostoski, H. R. Sadeghpour, and D. H. Santamore, Electric Noise Spectra of a Near-Surface Nitrogen-Vacancy Center in Diamond with a Protective Layer, Phys. Rev. Applied 10, 064056 (2018).

[47] J. T. Muhonen, J. P. Dehollain, A. Laucht, F. E. Hudson, R. Kalra, T. Sekiguchi, K. M. Itoh, D. N. Jamieson, J. C. McCallum, A. S. Dzurak, and A. Morello, Storing quantum information for 30 seconds in a nanoelectronic device, Nat. Nanotechnol. 9, 986 (2014).

[48] https://doi.org/10.5281/zenodo.4600786. 